\title{
PH-6
}

\section{Noninvasive light-reflection technique for measuring optical properties of human teeth}

\author{
Mohamed M.Gadallah' ${ }^{1,}$ Ashraf F. El-Sherif ${ }^{2,}$ Yasser H. El-Sharkawy ${ }^{3}$ \\ ${ }^{1,3}$ Biomedical Engineering Departments,MTC, Cairo, Egypt. \\ ${ }^{2}$ Laser Photonics Research Group, Engineering Physics Dept., MTC, Cairo, Egypt.
}

\begin{abstract}
The development of optical methods in modern medicine in the areas of diagnostics, therapy, and surgery has stimulated the investigation of optical properties of various biological tissues. The aim of this work is to investigate the feasibility of human teeth differentiation using laser, for this purpose, different laser sources have been used in order to compare their effect on the human teeth and consequently their accuracy in teeth differentiation .also, it is required to determine the type of laser that results in best accuracy. The experiment work in this research depends on using twenty human noncavitated caries lesion classified according to their class of caries. An ocean Blue HR2000 high resolution spectrometer is utilized to evaluate the optical properties of the teeth .the absorption coefficient are calculated under the effect of four excitation wavelengths (457 nm, $488 \mathrm{~nm}, 514 \mathrm{~nm}$, and $633 \mathrm{~nm}$ ).
\end{abstract}

The results have showed that the argon laser at $488 \mathrm{~nm}$ is more sensitive for carious detection and it is the most suitable for human teeth ablation.

Keywords: human caries detection, optical properties of human teeth, spectroscopy

\section{INTRODUCTION}

The optical properties of a tissue affect both diagnostic and therapeutic applications of light. The ability of light to penetrate a tissue, interrogate the tissue components, and then escape the tissue for detection is the key for diagnostic applications. The ability of light to penetrate a tissue and deposit energy via the optical absorption properties of the tissue is the key for therapeutic applications. Hence, specifying the optical properties of a tissue is the first step toward properly designing devices, interpreting diagnostic measurements or planning therapeutic protocols. The second step is to use the optical properties in a light transport model to predict the light distribution and energy deposition.

The light propagation in turbid biological media is governed by both its absorption and scattering properties. This type of spectroscopic measurements can provide information in many different scientific fields including medicine. In medicine, the knowledge of tissue optical properties has found its application in cancer diagnostics and therapy. The changes of optical properties by laser treatments are particularly interesting [1, 2]. Light propagations, absorption and scattering, changes by the kinetic changes of optical properties in laser irradiations. This problem is clinically very important for the realization of safe laser 
Military Technical College

Kobry Elkobbah,

Cairo, Egypt

April 19-21,2016

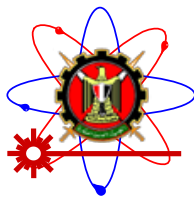

$8^{\text {th }}$ International Conference on

Mathematics and Engineering

Physics (ICMEP-8)

treatments because the understandings of optical properties by several laser parameters can realize the pre-estimated treatment effects.

In laser medicine, the accurate knowledge about the optical properties (scattering coefficient: $\mu$ s, absorption coefficient: $\mu$ s, scattering anisotropy: $\boldsymbol{g}$ ) of target tissue is important for the understanding prediction of propagation and distribution of light in tissues. [3]

In this work, these properties of human teeth and carious tissues is of great importance for interpretation and quantification of the diagnostic data, and for prediction of light distribution and absorbed energy for therapeutic and surgical use. There are some methods used in detecting optical properties. The most common technique for extracting the optical properties of tissue in vitro is the Integrating Sphere (IS) method, Double integrating sphere system [48] with an intervening sample is designed for non-invasive measurement of the optical properties of biological tissues, On the other hand, Inverse Monte Carlo methods [9-11] can be used to determine the optical properties separately over broad spectral range, without limitation of their accuracy.

In this paper, the optical absorption coefficient, $\mu \mathrm{a}$, in terms of the average human teeth were calculated using optical spectroscopy for different commercial law power laser source at wavelengths $457 \mathrm{~nm}, 488 \mathrm{~nm}, 514 \mathrm{~nm}$ and $633 \mathrm{~nm}$, and calculate the ability of laser to penetrate a human teeth and deposit energy to determine the maximum absorption coefficient for carious region and minimum for normal one (laser sensitivity).

\section{THEORETI CAL background}

Methods for determining the optical parameters of tissues can be divided into two large groups, direct and indirect methods. One of the indirect methods to determine optical properties of tissues. Spell out acronyms unless they are widely known the optical response of tissue

If tissue is irradiated with a laser beam, the fluence rate $\left[\mathrm{W} / \mathrm{m}^{2}\right]$ of the beam inside the tissue decreases exponentially (beer's law).the attenuation of the laser beam is due to absorption and possibly scattering of the collimated light according to the relation

$$
E(z)=\left(1-r_{S}\right) E_{\diamond} e_{t}^{-\mu z}
$$

$\mathrm{E}(\mathrm{z})$ is the attenuated beam within the tissue $[\mathrm{W} / \mathrm{m}]$.

$\mathrm{E} \circ$ is the irradiance $[\mathrm{W} / \mathrm{m}]$.

$\mu_{\mathrm{t}}$ is the attenuation coefficient. $[1 / \mathrm{m}]$

$r_{s}$ is the Fresnel specular reflection for nonpolarized light is about $2 \%$ for light normal to an air tissue interface.

Laser -tissue interaction is viewed as the propagation of photons through a volume of randomly distributed absorption and scattering centers.in the framework of this representation, the probability that a photon will be absorbed or scattered over the distance $\Delta \mathrm{s}$ is given by $\mu_{\mathrm{t}} \Delta \mathrm{s}$. Further, the probability that the photon is absorbed over the distance $\Delta \mathrm{s}$ is $\mu_{\mathrm{a}} \Delta \mathrm{s}$ where $\mu \mathrm{a}$ is the absorption coefficient with units $[1 / \mathrm{m}]$.likewise, it follows that the probability of a scattering event over $\Delta \mathrm{s}$ is $\mu_{\mathrm{s}} \Delta \mathrm{s}$ where $\mu_{\mathrm{s}}$ is the scattering coefficient with units $[1 / \mathrm{m}]$.based on these definitions and assuming that absorption and scattering are disjoint event. 


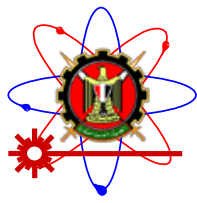

\subsection{Optical Properties of Tissue}

To understand the various modalities of laser-tissue interaction, it is necessary to get an overview of how photons penetrate biological tissue and how physics of absorption and scattering. (Fig.1).

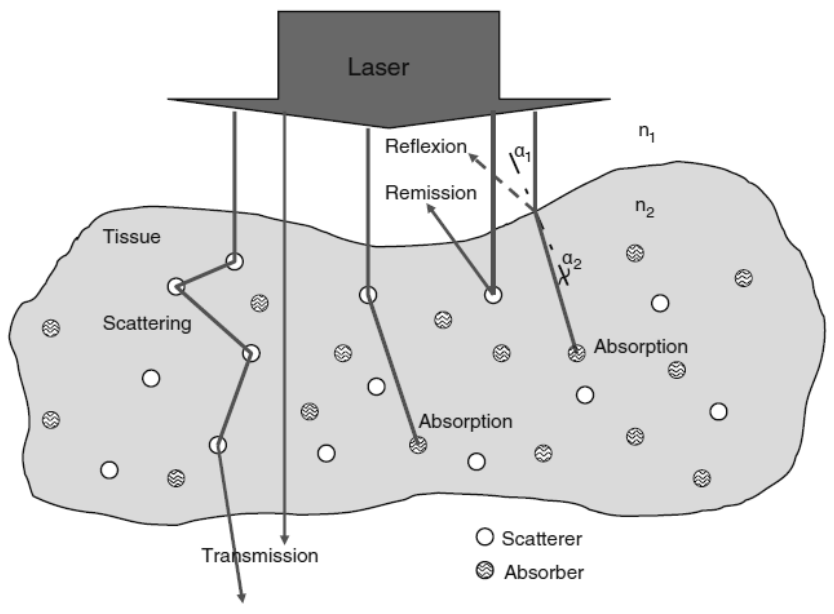

Fig.1 the various modalities of laser-tissue interaction

\subsection{Absorption and penetration}

The energy states of molecules are quantized; therefore, absorption of a photon takes place only when its energy, $\mathrm{E}=\mathrm{hv}$, corresponds to the energy difference between such quantized states. Absorption of a photon by a chromophore causes either a quantized change in the distance between charges (electron transition, ultraviolet or visible spectrum) or a quantized change of vibrational modes of the molecule (vibration transition, near infrared [NIR]).

Where electronic transitions are excited, leading to discrete and intense (broad) absorption bands. In the NIR and mid-infrared (MIR) region, tissue absorption is dominated by water absorption, with the maximum at $3 \mu \mathrm{m}$. The co efficient $\mu_{\mathrm{a}}\left(\mathrm{cm}^{\wedge}(-1)\right)$. Characterizes the absorption. The inverse $l_{a}$ defines the penetration depth (mean free path) into the absorbing medium.

\subsection{Scattering}

The scattering behavior of biological tissue is also important because it determines the volume distribution of light intensity in the tissue. This is the primary step for tissue interaction, which is followed by absorption and heat generation. Scattering of a photon is accompanied by a change in the propagation direction without loss of energy. Scattering structures of the tissue can be macroscopic like muscle fibers, skin layers, or dentin tubules; microscopic like cells or intracellular structures; and even sub-microscopic, taking into account macromolecules or nanoparticles

According to the size of the scattering structure, one has to distinguish between Rayleigh scattering, $\mathrm{d}<<\mathrm{l}$, and Mie scattering, $\mathrm{d} \geq 1$. Scattering of tissue is always a combination of Rayleigh and Mie scattering The scattering, similar to absorption, is expressed by the scattering coefficient $\mu_{\mathrm{s}}\left(\mathrm{cm}^{\wedge}(-1)\right)$. The inverse parameter, $1 / \mu_{\mathrm{s}}(\mathrm{cm})$, is the mean free path length until a next scattering event occurs.

We have seen from Mie scattering that scattering is not isotropic. Forward scattering is predominant in biological tissue. This characteristic is described by the anisotropy factor $\mathrm{g}$. $\mathrm{g}$ 
can have absolute values from 0 to 1 , from isotropic scattering $(\mathrm{g}=0)$ to forward scattering ( $\mathrm{g}$ $=1$ ). Negative values for $g$ stand for backward scattering. In tissue, g can vary from 0.8 to 0.99. Anisotropy factor $\mathrm{g}$ : $0 \leq \mathrm{g} \leq 1 \mathrm{~g}=0$ : isotropic scattering; $\mathrm{g}=1$ : forward scattering. Taking into account the g value, a reduced scattering coefficient, $\mu_{\mathrm{s}}^{\prime}\left(\mathrm{cm}^{\wedge}(-1)\right)$. , is defined as

$$
\mu_{\mathrm{s}}{ }^{\prime}=\mu_{\mathrm{s}}(1-\mathrm{g})
$$

In MCSs, one has to consider a probability function for $\mathrm{g}$ in what direction a photon is scattered. The Henyey-

Greenstein phase function [25] p $(\theta)$ is often used to describe the angular distribution of light scattered by tissue. It is characterized by the average $\operatorname{cosine}<\cos \theta>$ of the scattering angle $\theta$. Since the Henyey-Greenstein phase function is a probability density function, it is normalized to an area of 1 . This model has been applied to numerous situations, ranging from the scattering of light by biological tissue to scattering by interstellar dust clouds. The angular distribution of scattered light is given by

$$
\mathrm{P}(\theta)=1 / 4 \pi(1-\mathrm{g} \wedge 2) /(1+\mathrm{g} \wedge 2-2 \mathrm{~g} \cos (\theta))^{\wedge}(3 / 2)
$$

The parameter g characterizes the normalized distribution. For some values of g, shows the probability of scattering from $-180^{\circ} \leq \theta \leq+180^{\circ}$. When $\mathrm{g}$ approaches the value 1 , then the function is very peaked around $\theta=0$. In case of isotropic scattering, $g=0$, there is a constant value of $1 / 4 \pi$ overall scattering angles $\theta$.

The sum of $\mu_{\mathrm{s}}$ and $\mu_{\mathrm{a}}$ is called the total attenuation coefficient $\mu_{\mathrm{t}}(\mathrm{cm} \wedge(-1))$. :

$$
\mu_{\mathrm{t}}=\mu_{\mathrm{s}}+\mu_{\mathrm{a}}\left(\mathrm{cm}^{\wedge}(-1)\right)
$$

Measuring the optical constants of biological tissues is not a simple task. In a configuration where a collimated beam hits a tissue sample of defined thickness and only the transmitted photons (ballistic photons) reach the detector, the attenuation coefficient $\mu_{\mathrm{t}}$ can be measured. Some other expressions are helpful to describe the optical properties of biological tissue. The fraction of the scattering $\mu_{\mathrm{s}}$ over the total attenuation $\mu_{\mathrm{t}}$ is called Albedo a:

$$
\text { Albedo } \mathrm{a}=\mu_{\mathrm{s}} /\left(\left(\mu_{\mathrm{s}}+\mu_{\mathrm{a}}\right)\right)
$$

Whereas the effective damping coefficient $\mu$ eff is defined as

$$
\mu \mathrm{eff}=\sqrt{ }\left(\left(3 \mu_{\mathrm{s}}\left(\mu_{\mathrm{s}}+\mu_{\mathrm{a}}\right)\right)\right) \quad\left(\mathrm{cm}^{\wedge}(-1)\right)
$$

The inverse value $1 / \mu_{\text {eff, }}$ is called the effective penetration depth, $d_{\text {eff }}$ of light into tissue:

$$
D_{\text {eff }}=1 / \mu_{\text {eff }} \quad(\mathrm{cm})
$$

In this work, to differentiate between normal and carious parts of teeth, a sensitivity parameter is proposed. This sensitivity is defined as the ratio between the absorption coefficients of the carious $\left(\mu_{\mathrm{ac}}\right)$ and the absorption coefficient of the normal parts $\left(\mu_{\mathrm{an}}\right)$

$$
\text { Sentivity } \mathrm{s}=\mu_{\mathrm{ac}} / \mu_{\mathrm{an}}
$$

\section{METHODS and materials}

\subsection{Dental specimen selection}

Twenty teeth with natural noncavitated enamel carious lesions on smooth surfaces were selected from extracted human dental hospital. The lesions were classified clinically, by 
Military Technical College

Kobry Elkobbah,

Cairo, Egypt

April 19-21,2016

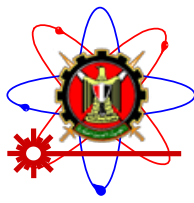

$8^{\text {th }}$ International Conference on

Mathematics and Engineering Physics (ICMEP-8)

dentists according to clinical judgment into classes as class1, 2 and 5.In this paper we examined teeth from class 2 .

\subsection{Experimental technique}

The experiment was set up to illuminate the teeth by four wavelengths $(457 \mathrm{~nm}, 488 \mathrm{~nm}$, $514 \mathrm{~nm}$, and $633 \mathrm{~nm}$ ) of laser sources, scan it, and measure the difference in absorption between four selected points starting from normal tissue up to the center of carious tissue for each teeth. The obtained absorption measures for the four points are compared to differentiate between the normal and carious tissue. This process is repeated for each of the four wavelengths. In these experiments, the (spectra suite spectrometer operating software is used to measure the absorption coefficients at the selected points).

First we scanned the teeth through four points we choose these points according to the difference in the grade of carious from normal to center of carious by four wavelengths of different types of laser (blue 457nm, argon 488nm,argon 514nm and He-NE 633nm)

Second, with scanning we put suitable detector backward of the teeth to detect the laser beam after hitting the teeth and connect the detector to the suitable spectrometer (NIR- 4000 SPECTROMETER), By using (SpectraSuite Spectrometer Operating Software) we can determine the Absorption coefficient and we can draw four graphs for each wavelength which indicate the difference in Absorption coefficient of each point according to the different types of laser.

Finally, we calculate the effective transport coefficient and effective length (penetration depth) for each type of laser in order to choose the best wavelength in differentiating between caries and normal tissue according to best sensitivity.

For determination of these coefficients we measured the optical properties of each tooth with a specific detector (fiber optical cable) to A NIR-4000 is spectrophotometer. Four wavelengths $(457 \mathrm{~nm}, 488 \mathrm{~nm}, 514 \mathrm{~nm}$, and $633 \mathrm{~nm}$ ) pulsed from three laser source, first is blue laser $457 \mathrm{~nm}$, second is argon $488 \mathrm{~nm}$, third is argon $514 \mathrm{~nm}$, and fourth is He-NE 633nm. Initially, the tip of laser source was directed into an empty sample holder in a darkened room and a background spectrum was recorded, which was subsequently subtracted from each reading. These tests were undertaken to ensure that the spectra recorded from dental samples were due to the sample and not due to materials used in the fabrication of the optical fibers used in the probe. The laser was activated and the output from the tip of laser source directed onto a sample and the optical fiber probe was put in back of the dental sample to detect the laser beam after hitting the sample and analyzed in the spectrometer. Some several peaks were noticed but as these peaks were outside the spectral region of interest used for the later analysis and were therefore ignored.

We were thus certain that any spectral features observed were due to components present in the dental samples. Normalization was undertaken by first subtracting from each spectral point the average background. The spectra were stored on a PC and analyzed using computer SpectraSuite Spectrometer Operating Software

\section{DI SCUSSI ON and results}

The most important factor for caries detection, subsequent diagnosis and treatment planning, is to determine the extent of a lesion and to monitor the course of the disease in a quantitative and noninvasive manner. Caries can be described as a process resulting in structural changes to the dental hard tissue. The Diffusion of calcium, phosphate, and carbonate out of the tooth, the demineralization process, will result in loss of mineral content. Most of the optical 


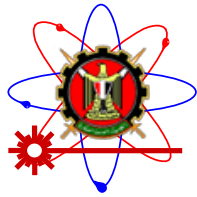

\section{$8^{\text {th }}$ International Conference on Mathematics and Engineering Physics (ICMEP-8)}

methods are based on the differences in light scattering, absorbing or Fluorescence between the sound enamel and the lesion.

Absorption is the process in which photons are stopped by an object and the wave energy is taken in by the object. The energy lost is mostly converted into heat or into another wave which has less energy and hence longer wavelengths. Absorption of a photon takes place only when its energy, $E=h v$, corresponds to the energy difference between such quantized states.

In this experiment we depended on absorption process calculating the absorption coefficient of each point which we selected with each wavelength used in this experiment.

First we scanned the teeth through four points we choose these points according to the difference in the grade of carious from normal to center of carious as shown in the left side of (Fig.2) by four wavelengths of different types of laser (blue 457nm,argon 488nm,argon 514nm and He-NE 633nm)

Second, with scanning we put suitable detector backward of the teeth to detect the laser beam after hitting the teeth and connect the detector to the suitable spectrometer (NIR- 4000 SPECTROMETER), By using (SpectraSuite Spectrometer Operating Software) we can determine the Absorption coefficient and we can draw four graphs for each wavelength which indicate the difference in Absorption coefficient of each point according to the different types of laser as shown in the middle of (Fig.2).

Finally, from each graph we draw relationship between absorption coefficient and the points which chosen as shown in the right of (Fig.2) to calculate the sensitivity for each wavelength to compare between them and chose best one in differentiating between normal and carious part.

$$
\text { Carious }
$$

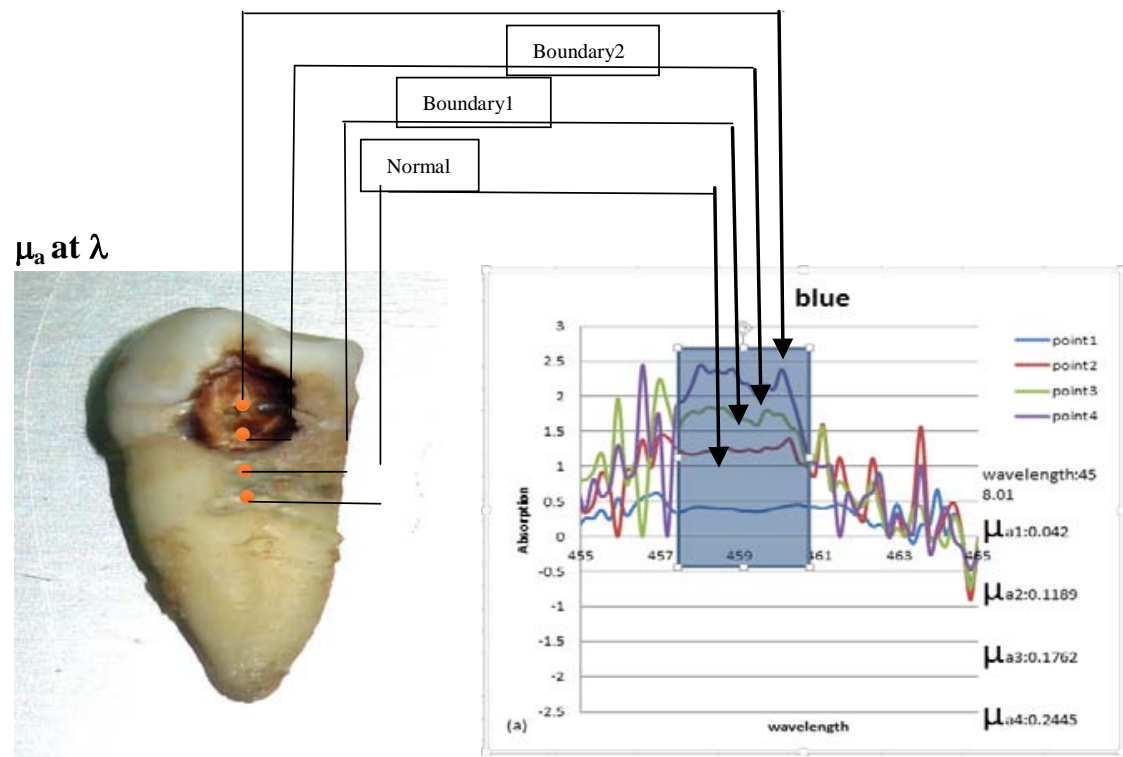

Absorption Coef.

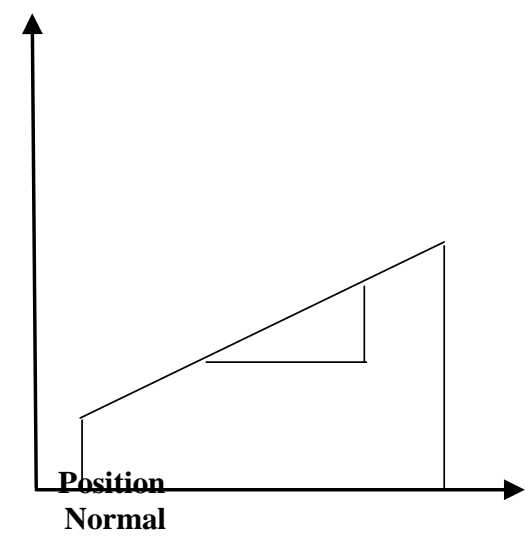

Carious

Fig2. (a) Scanning teeth by blue laser, (b) absorption coefficients values and (c) general sensitivity curve

SpectraSuite Spectrometer Operating Software calculates Absorption coefficient using the following equation. 
Military Technical College

Kobry Elkobbah,

Cairo, Egypt

April 19-21,2016

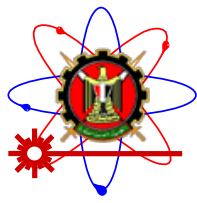

$8^{\text {th }}$ International Conference on Mathematics and Engineering Physics (ICMEP-8)

$$
A=-\log 10\left(\left(S_{\lambda}-D_{\lambda}\right) \backslash\left(R_{\lambda}-D_{\lambda}\right)\right)
$$

Where:

$\mathrm{S}=$ Sample intensity at wavelength $\lambda$

$\mathrm{D}=$ Dark intensity at wavelength $\lambda$

$\mathrm{R}=$ Reference intensity at wavelength $\lambda$

By using spectrasuite software we can analyze the data and indicate the difference in absorption coefficients for each wavelength through the selected four points as shown in (Fig.3)
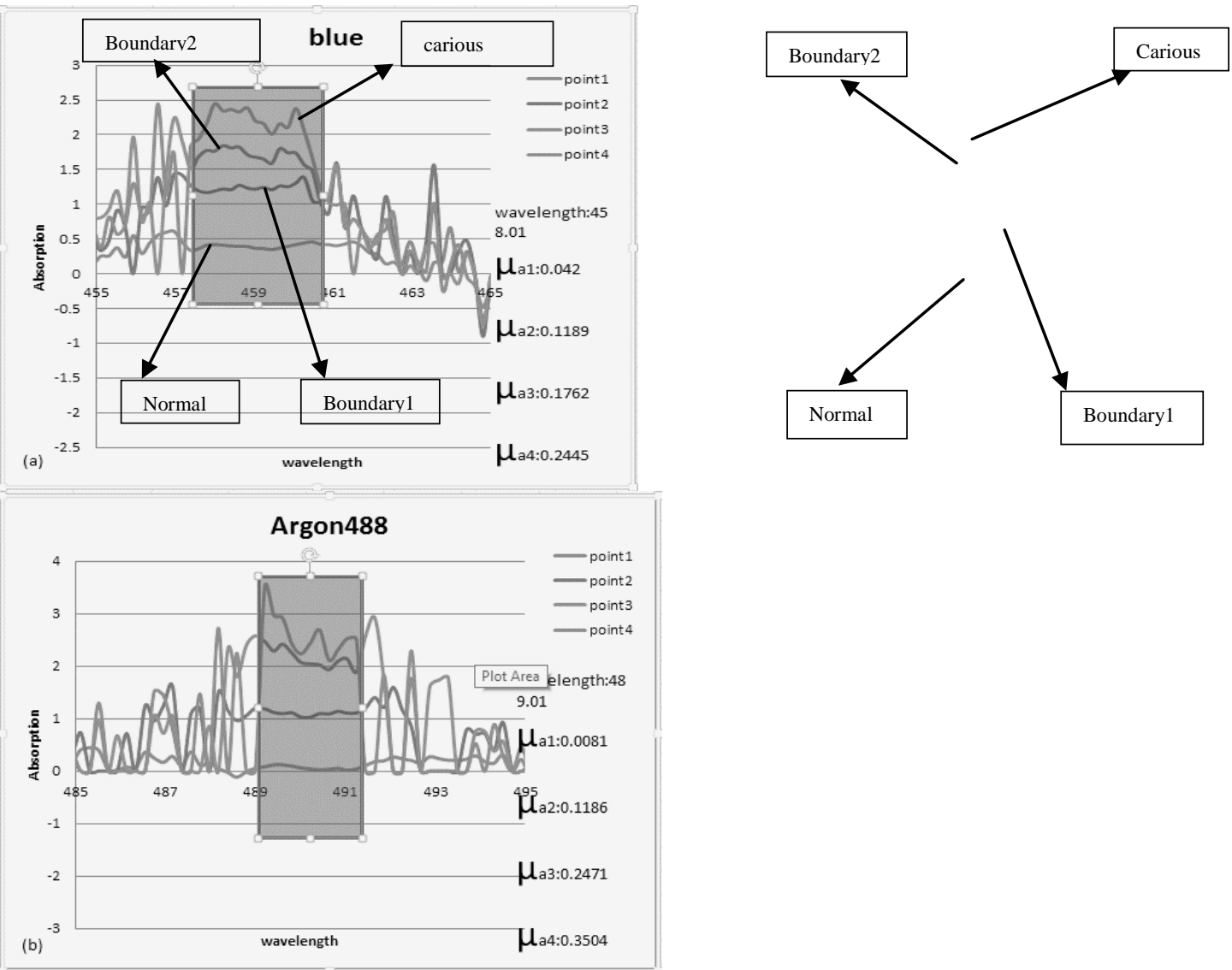
Military Technical College

Kobry Elkobbah,

Cairo, Egypt

April 19-21,2016

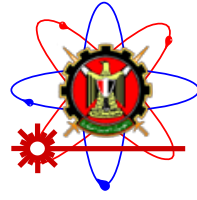

$8^{\text {th }}$ International Conference on Mathematics and Engineering Physics (ICMEP-8)
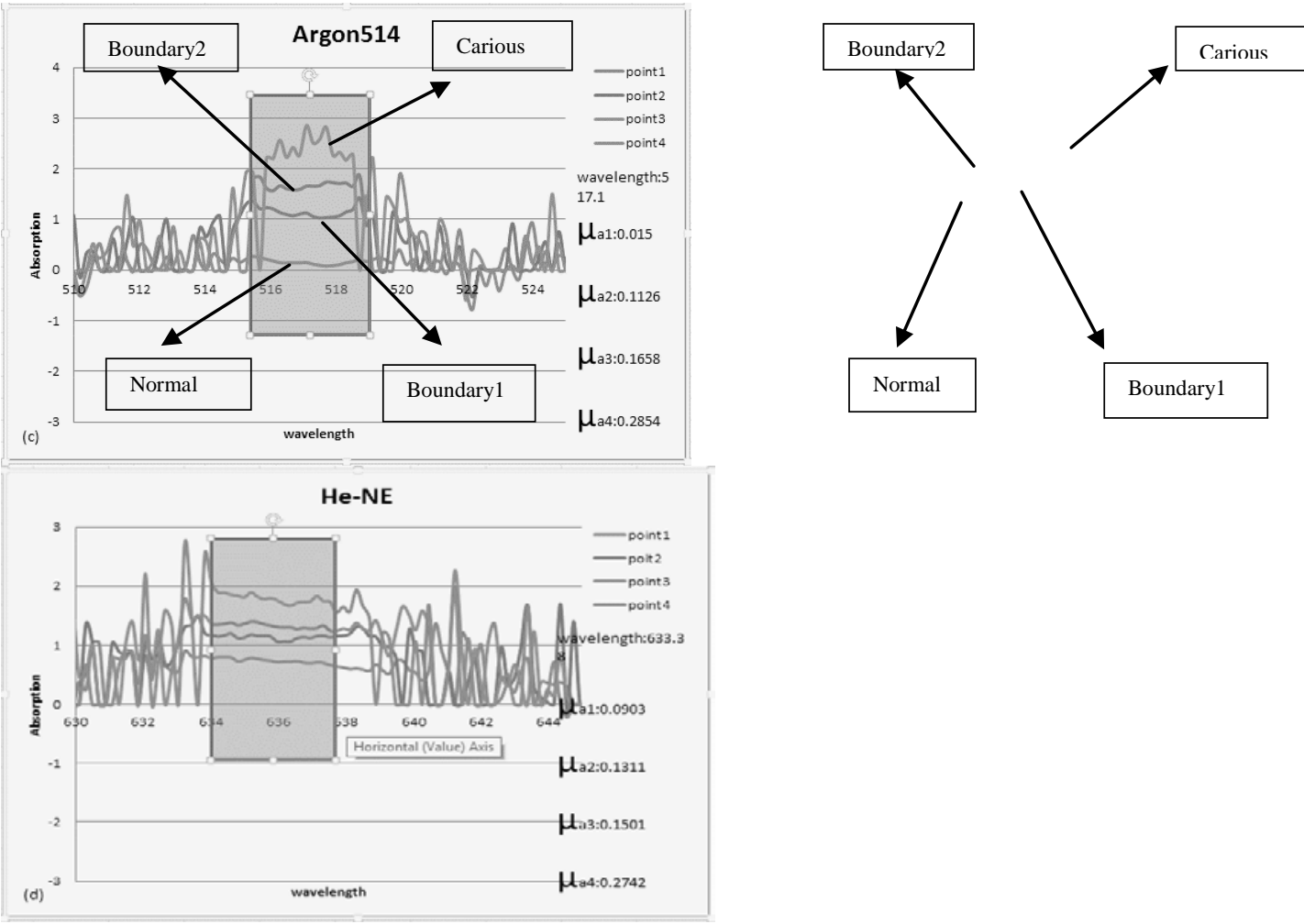

Fig.3 (a) absorption of blue laser 457nm measured by spectrometer of four position in the teeth, (b) absorption of argon laser 488nm measured by spectrometer of four position in the teeth, (c) absorption of blue laser 514nm measured by spectrometer of four position in the teeth, (d) absorption of He-NE laser 633nm measured by spectrometer of four position in the teeth

From these graphs we measured the absorption coefficients for each point in the sample with all wavelengths as shown result in table1

Table .1 Measured absorption coefficient for scanned decayed human teeth at four positions.

Coefficient of absorption

\begin{tabular}{ccccc}
\hline \multirow{2}{*}{ Bands } & Sound & \multicolumn{2}{c}{ Edge of caries } & Caries \\
\cline { 2 - 5 } & $\begin{array}{c}\text { Point1(normal } \\
\text { tissue) }\end{array}$ & $\begin{array}{c}\text { Point2(boundary } \\
\text { area) }\end{array}$ & $\begin{array}{c}\text { Point3(boundary } \\
\text { area) }\end{array}$ & $\begin{array}{c}\text { Point4(severed } \\
\text { decayed tissue) }\end{array}$ \\
\cline { 2 - 5 } 457 & 0.042 & 0.1189 & 0.1762 & 0.2445 \\
488 & 0.0081 & 0.1186 & 0.2471 & 0.3504 \\
514 & 0.015 & 0.1126 & 0.1658 & 0.2854 \\
633 & 0.0903 & 0.1311 & 0.1501 & 0.2742
\end{tabular}



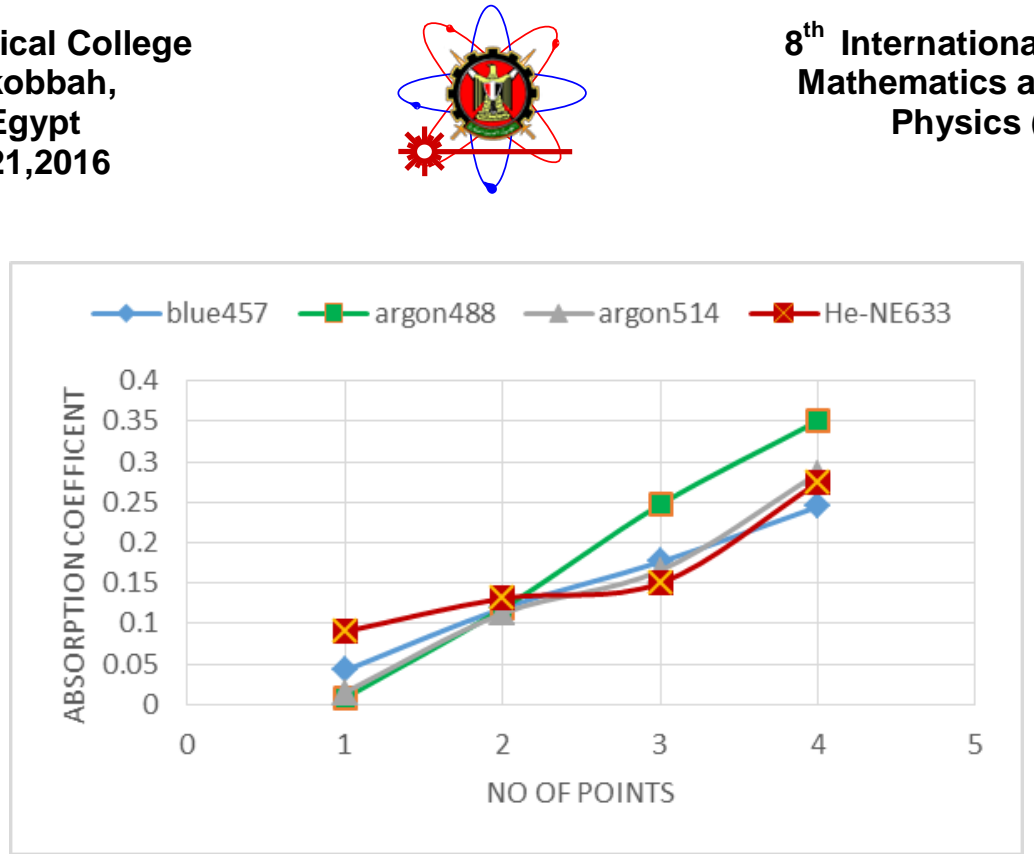

Fig.4 plot of absorption coefficients for each point with four wavelengths

From above table(1) and according to laws that explained in the theoretical background we can summarize some optical properties in the followed table and after making curve fitting as shown in (Fig.4) we can calculate the sensitivity for each wavelength in detection the caries and differentiate between normal and caries region.

the importance of the sensitivity is to differentiate between four types of laser and which one is the best to detect any change in the structure of the teeth (i.e. from normal and carious) We can draw a relationship between absorption coefficient and each position in the sample for each wavelength then we make a curve fitting by MATLAB to calculate sensitivity this result shown in (Fig.5). 
Military Technical College

Kobry Elkobbah,

Cairo, Egypt

April 19-21,2016 $8^{\text {th }}$ International Conference on Mathematics and Engineering Physics (ICMEP-8)

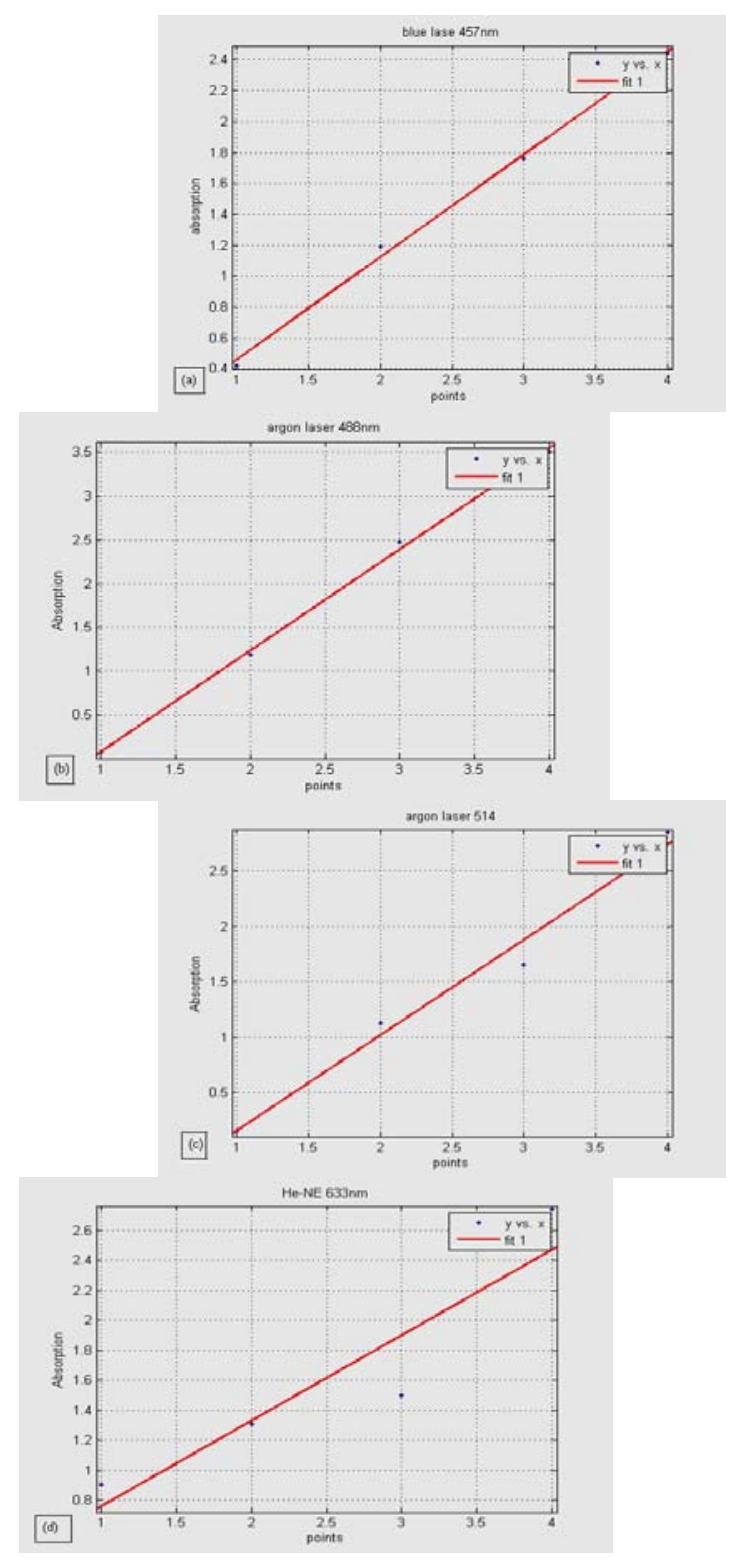

Fig.5 (a) blue laser sensitivity 457nm, (b) argon laser sensitivity 488nm, (c) argon laser sensitivity 514nm, (d) He-NE laser sensitivity 633nm

Table .2 Calculated optical properties coefficients for carious areas of human teeth

\begin{tabular}{|c|c|c|c|}
\hline$\lambda(\mathrm{nm})$ & $\mu_{a}\left(\mathrm{~cm}^{-1}\right) \approx \cdot \mu_{\text {eff }}$ & $l_{\text {eff }}(\mathrm{cm})$ & Sensitivity \\
\hline 457 & 2.445 & 0.4089 & 0.8644 \\
\hline 488 & 3.504 & 0.2853 & 1.155 \\
\hline 514 & 2.854 & 0.3503 & 0.6648 \\
\hline 633 & 2.742 & 0.3646 & 0.5707 \\
\hline
\end{tabular}


Military Technical College

Kobry Elkobbah,

Cairo, Egypt

April 19-21,2016

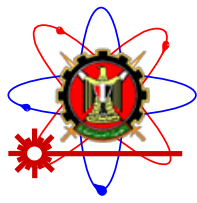

$8^{\text {th }}$ International Conference on

Mathematics and Engineering

Physics (ICMEP-8)

For each wavelength, the values of the optical properties determined are presented in Tables (2). These tables show the experimental values of: the absorption coefficient $\mu_{a}$, and the effective mean free path $\mathrm{L}_{\text {eff. }}$. As pointed out above we depended on the absorption parameter and absorption is the dominant property in carious part that we can say $\mu_{\mathrm{a}} \approx \mu$ eff , and $L_{\text {eff }}$ values were calculated as indicated in equation (7). On the other hand, the data show that Argon $488 \mathrm{~nm}$ has best sensitivity (0.8644) which mean that it's best wavelength to differentiate between carious and normal tissue, the second wavelength is blue $457 \mathrm{~nm}$ has sensitivity (0.8644) which mean it can also use in differentiate between normal and carious tissue.

We find in previous work that three types of these wavelengths already used in diagnostics in dentistry Argon 488nm,argon 514nm and He-NE 633nm[13]. From measurement we found blue laser457nm can be used in differentiation between normal and carious tissue. Another advantage from results that effective length for blue laser is the highest which mean that it can be used removing carious or ablation.

\section{5. conclusion}

This We believe that this overview of tissue optical properties will provide users a possibility to predict optical properties of tissues under their interest and evaluate light distribution in the organ under examination or treatment Optical properties of human teeth, at (Argon $488 \mathrm{~nm}$, blue $457 \mathrm{~nm}$, Argon $514 \mathrm{~nm}$ and He-NE $633 \mathrm{~nm}$ ) have been determined using the spectrometer. We generally observed that the measured diffuse reflectance and transmittance of human teeth (normal and decayed tissues) decrease in reflectance, scattering, while absorption coefficients increased at $514 \mathrm{~nm}$. These results of our study yield an overall high differentiation potential for various laser source types after interact with human teeth, performing that the maximum absorption coefficient at wavelength $514 \mathrm{~nm}$, producing a powerful tools for carious detection and treatments.

\section{REFERENCES}

[1] Ritz, J.-P., Roggan, A., Isbert, C., Muller, G., Buhr, H. J., Germer, C.-T., "Optical properties of native and coagulated porcine liver tissue between 400 and $2400 \mathrm{~nm}$ ", Lasers Surg. Med. 29, 205-212 (2001).Davis, A. R., Bush, C., Harvey, J. C. and Foley, M. F., "Fresnel lenses in rear projection displays," SID Int. Symp. Digest Tech. Papers 32(1), 934-937 (2001).

[2] Yaroslavsky, A. N., Schulze, P. C., Yaroslavsky, I. V., Schober, R., Ulrich, F., Schwarzmaier, H.-J., "Optical properties of selected native and coagulated human brain tissues in vitro in the visible and near infrared spectral range”, Phys. Med. Biol. 47, 2059-2073 (2002).

[3] Katsunori Ishii, Akinori Kimura, Kunio Awazu "Optical Properties of Tissues after Laser Treatments in the Wavelength Range of 350 - 1000 nm” Proc. of SPIE Vol. 6991 69912F-6Jones, C. J., Director, Miscellaneous Optics Corporation, interview, Sept. 23 2011

[4] Pickering, J. W., Prahl, S. A., Wieringen, N. v., Beek, J. F., Sterenborg, H. J. C. M., Gemert, M. J. C. v., "Double integrating-sphere system for measuring the optical properties of tissue”, Appl. Opt. 32(4), 399-410 (1993). 


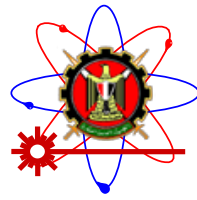

\section{$8^{\text {th }}$ International Conference on Mathematics and Engineering Physics (ICMEP-8)}

[5] Vries, G. d., Beek, J. F., Lucassen, G. W., Gemert, M. J. C. v., "The effect of light losses in double integrating spheres on optical properties estimation”, IEEE J. Select. Top. Quantum Electron. 5(4), 944-947 (1993).

[6] Roggan, A., Friebel, M., Dorschel, K., Hahn, A., Muller, G., "Optical properties of circulating human blood in the wavelength range 400-2500 nm”, J. Biomed. Opt. 4(1), 36-46 (1999).

[7] Troy, T. L., Thennadil, S. N., "Optical properties of human skin in the near infrared wavelength range of 1000 to $2200 \mathrm{~nm}^{\prime \prime}$, J. Biomed. Opt. 6(2), 167-176 (2001).

[8] Wei, H.-J., Xing, D., Wu, G.-Y., Gu, H.-M., Lu, J.-J., Jin, Y., Li, X.-Y., "Differences in optical properties between healthy and pathological human colon tissues using a Ti: sapphire laser: an in vitro study using the Monte Carlo inversion technique”, J. Biomed. Opt 10(4), 044022; 1-8 (2005).

[9] Meinke, M., Muller, G., Helfmann, J., Friebel, M., "Optical properties of platelets and blood plasma and their influence on the optical behavior of whole blood in the visible to near infrared wavelength range”, J. Biomed. Opt.12 (1), 014024; 1-9 (2007).

[10] Friebel, M., Roggan, A., Muller, G., Meinke, M., "Determination of optical properties of human blood in the Spectral range 250-1100 nm using Monte Carlo simulations with hematocrit-dependent effective scattering phase Functions”, J. Biomed. Opt. 11(3), 034021; 1-10 (2006).

[11] Salomatina, E. Jiang, B.Novak, J.Yaroslavsky, A. N., "Optical properties of normal and cancerous human skin in The visible and near-infrared spectral range”, J. Biomed. Opt. 11(6), 064026; 1-9 (2006).

[12] Henyey LG, Greenstein JL. Diffuse radiation in the galaxy. Astrophys J. 1941; 93:70-83.

[13] LJ Walsh“The current status of laser applications in dentistry” Australian Dental Journal; $48:$ : (3):146-155(2003). 